\title{
9. Cirugía cardiovascular
}

\subsection{Mieloma múltiple incidental en cirugía cardiaca}

Herrera Romero Gabriel Amadeus,* Herrera Camacho Gabriel,ł Flores Figueroa Fernando, Camacho Álvarez Bruno Bismarck, $\ddagger$ Gómez Ontiveros J Ignacio, $¥$ Nájar López Sergio,* Victal Adame Octavio

* Hospital de Especialidades San Francisco de Asís.

‡ Centro Médico Nacional de Occidente.

Tipo de estudio: Reporte de casos clínicos con revisión de fuentes

Introducción: El mieloma múltiple (MM) es una proliferación clonal de células plasmáticas con producción de inmunoglobulinas monoclonales. Su diagnóstico puede sospecharse por la presencia de diversas manifestaciones clínicas, entre ellas, la insuficiencia mitral secundaria a ruptura de cuerdas tendinosas es de las menos frecuentes. Descripción del caso: Presentamos un paciente el cual fue diagnosticado con insuficiencia mitral y, al realizar esternotomía, se aprecia tejido de aspecto mixoide, fue enviado a patología y se identificó plasmocitoma preplasmocítico. Metodología: Retrospectivo. Resultados: En la actualidad, pocos son los casos que hacen referencia de insuficiencia mitral causada por mieloma múltiple; en artículos analizados se reporta como es que el mieloma múltiple inicialmente diagnosticado es el que causa la insuficiencia mitral, clínicamente con los signos y síntomas que ya conocemos (pérdida de peso, alteraciones hematológicas como anemia, leucopenia, infecciones recurrentes, alteraciones renales, etcétera). Discusión: Es interesante cómo la mayoría de patologías oncológicas al inicio causan manifestaciones clínicas inespecíficas, sólo al momento de estar en estadios avanzados comienzan a causar alteraciones específicas, en el caso del mieloma múltiple la radiografía característica de cráneo en «sacabocado y sal y pimienta», aunque en otros casos como el presentado fue la insuficiencia mitral secundario a la invasión.

\subsection{Correlación de requerimiento transfusional intraoperatoria con variables quirúrgicas}

Cárdenas Chávez Jesús Israel, Carrasco Castilla Alma Ivette, García Molina Ixchel

Hospital de Cardiología del Centro Médico Nacional Siglo XXI.

Tipo de estudio: Estudio pronóstico

Introducción: Características propias de la cirugía derivan en anemia, siendo casi el $80 \%$ de los pacientes los que ameritarán transfusión durante o después del evento. La cirugía cardiovascular corresponde al 10-15\% de la donación. La transfusión como factor de riesgo independiente para la mortalidad es controversial; en conjunto con variables como edad, comorbilidades, sangrado y reintervenciones influyen en el desenlace. Objetivo: Analizar la correlación entre la cantidad de transfusión de CE con las principales variables quirúrgicas en pacientes sometidos a cirugía cardiovascular en el Hospital de Cardiología del Centro Médico Nacional Siglo XXI. Metodología: Estudio transversal analítico. Se incluyeron pacientes sometidos a cirugía cardiovascular en el año 2017. Se registraron las características clínicas, transfusionales, bioquímicas y se realizó análisis de correlación con prueba de Pearson para determinar la mortalidad. Resultados: Se analizaron 399 pacientes; la cirugía más frecuente IVM 82\% (329), seguido de
Tabla 9.2.1: Correlación de Pearson, ante variables quirúrgicas y variables transfusional.

\begin{tabular}{lcc} 
Variables & $\begin{array}{c}\text { Coeficiente } \\
\text { de correlación }\end{array}$ & $\begin{array}{c}\mathbf{p} \\
\mathbf{a}=5 \%\end{array}$ \\
\hline Edad/sangrado & 0.053127868 & $<0.05$ \\
Edad/transfusiones & -0.088123159 & $<0.05$ \\
Sangrado/DCP & 0.140121139 & $<0.05$ \\
Sangrado/TI & 0.06343508 & $<0.05$ \\
DCP/Hb postquirúrgica & 0.016400832 & $<0.05$ \\
CE transfundido/Hb postquirúrgica & -0.057503967 & $<0.05$ \\
\hline
\end{tabular}

$\mathrm{DCP}$ = derivación cardiopulmonar; $\mathrm{TI}$ = tiempo de isquemia;

$\mathrm{Hb}=$ hemoglobina; $\mathrm{CE}$ = concentrado eritrocitario.

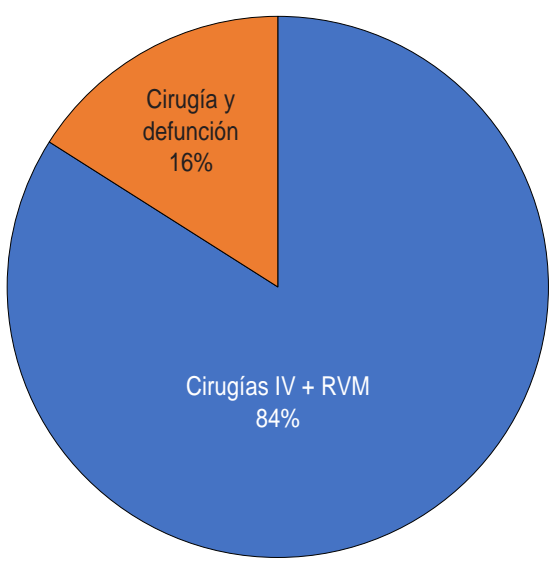

Figura 9.2.1: Cirugías «implante valvular y revascularización» en el aก̃o 2017 ( $N=399)$.

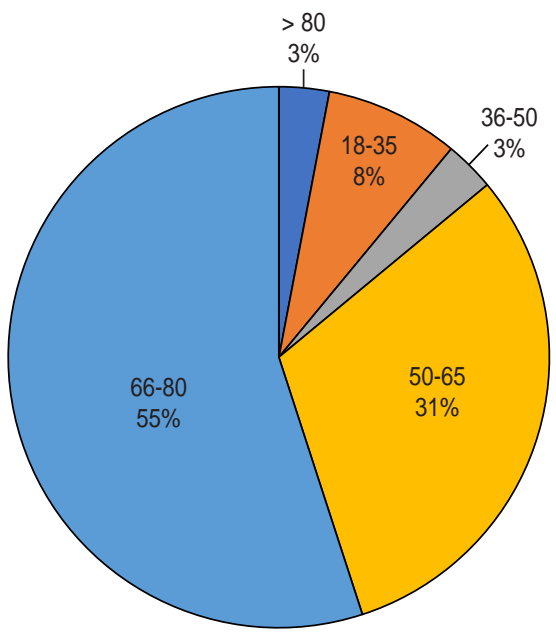

Figura 9.2.2: Defunciones por rango de edad $(N=68)$. 
RVM 15\% (62). El 15\% (62) fueron defunciones, con el 4\% durante reintervención quirúrgica; el género masculino predominó con el 54\% (34). Rango de edad más frecuente fue 66-80 años de edad. Existió una correlación directa: mayor edad, mayor requerimiento transfusional intraoperatorio; y una correlación inversa: menor transfusión, mayores cifras de hemoglobina postquirúrgicas. Discusión y conclusiones: Tener una evaluación preoperatoria interdisciplinaria de los requerimientos transfusionales de cada paciente permitirá una preparación clínica adecuada antes de cada intervención quirúrgica, aumentando la eficacia en el pronóstico y tratamiento.

\subsection{Criterios de transfusión masiva más frecuentes en pacientes sometidos a cirugía cardiovascular}

Roa Gómez Lesley, Jiménez Salazar Marco Antonio,

Neri Rodríguez Jorge Luis

Hospital de Cardiología del Centro Médico Nacional Siglo XXI.

Tipo de estudio: Registros locales o nacionales

Introducción: La transfusión de sangre y sus componentes es una modalidad terapéutica esencial en la práctica médica. Este procedimiento conlleva riesgos y complicaciones, sobre todo si se convierte en transfusión masiva, un remplazo de grandes volúmenes de sangre en periodo corto de tiempo. La cirugía cardiaca es el escenario más común para la transfusión masiva. Objetivo: Conocer los diferentes criterios de transfusión masiva descritos en la literatura. Establecer la frecuencia de transfusión masiva en el Hospital de Cardiología del Centro Médico Nacional Siglo XXI de 2018 a 2019. Metodología: Analizamos 730 registros clínicos de hemovigilancia de pacientes hospitalizados en el Hospital de Cardiología del Centro Médico Nacional Siglo XXI durante 2018 y 2019. Se seleccionaron a los pacientes reportados como transfusión masiva para la identificación de los criterios más utilizados para este diagnóstico, y determinar su frecuencia acorde con las definiciones descritas en la literatura. Resultados: Durante 2018 y 2019 se registraron 44 transfusiones masivas, los criterios más utilizados para definirla fueron la reposición de una volemia completa en 24 horas (47.7\%), seguida de transfusión de cuatro concentrados eritrocitarios en una hora (34\%), reposición del 50\% de la volemia en tres horas (15.9\%) y finalmente transfusión de 10 concentrados eritrocitarios en 24 horas (2.3\%). Discusión y conclusiones: Los criterios más considerados en la definición de transfusión masiva son los descritos en la Norma Oficial Mexicana NOM-003-SSA2-1993, seguidos de la literatura internacional para protocolo de transfusión masiva.

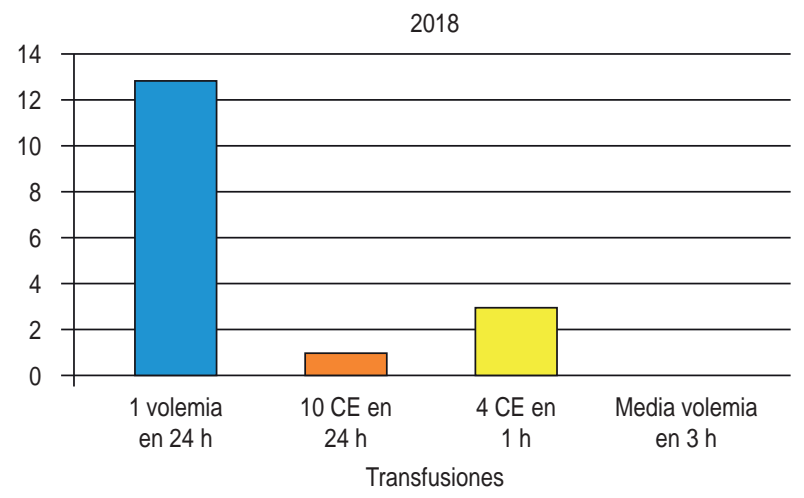

Figura 9.3.1.

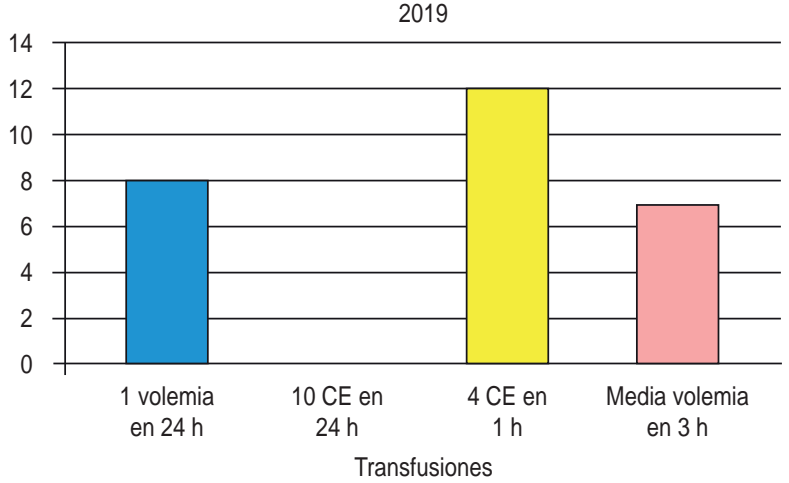

Figura 9.3.2.

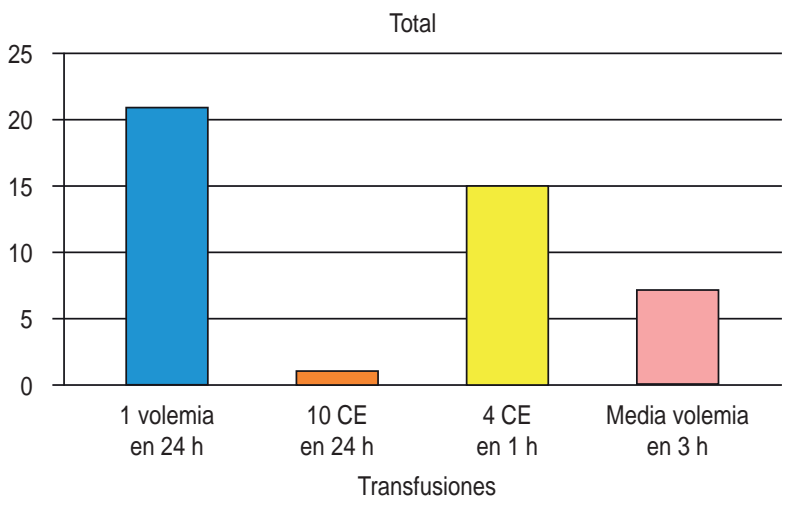

Figura 9.3.3.

\subsection{Perfil de laboratorio de pacientes cardiovasculares bajo oxigenación por membrana extracorpórea ECMO}

Fajardo González Luis Gerardo, Borja Rosas Betania Melina, Guevara Cedeño Gabriela Alejandra, García González Yair UMAE Hospital de Cardiología del Centro Médico Nacional Siglo XXI. Tipo de estudio: Prueba diagnóstica

Introducción: La oxigenación por membrana extracorpórea $(E C M O)$ es una modalidad de tratamiento para proporcionar soporte circulatorio cardiaco o respiratorio prolongado temporal para pacientes críticos. Las causas de muerte varían desde eventos cardiovasculares hasta disfunción orgánica, incluyendo fallas sistémicas asociadas con esta terapia. La identificación de los parámetros de riesgo es útil para la evaluación de estos pacientes. Objetivo: Describir el perfil de laboratorio durante la terapia con ECMO en pacientes cardiovasculares y observar las diferencias entre los pacientes que sobrevivieron a la terapia contra los que fallecieron. Metodología: Se realizó un estudio observacional longitudinal retrospectivo, se incluyeron 20 pacientes cardiológicos, recabando el histórico de estudios de laboratorio realizados durante el uso de ECMO de abril 2018 a mayo 2019 observándose las diferencias en pediátricos y adultos supervivientes y difuntos tras el uso de esta terapia; se utilizaron t de Student y $\chi^{2}$ para comparación. Resulta- 
dos: Las patologías reportadas fueron valvulopatías en 35\% ( $\mathrm{n}=$ 7), SICA-IAM 15\% $(n=3)$, malformaciones congénitas 15\% ( $n=$ 3) y otras malformaciones $20 \%(n=4)$. Se observó una diferencia significativa en niveles de creatinina de pacientes con ECMO que murieron durante la terapia respecto a supervivientes tanto adultos $(p=0.04)$ como pediátricos $(p=0.03)$, así como en niveles de urea en ambos grupos. Adicionalmente, se observó una diferencia significativa en los valores de plaquetas entre el grupo de supervivientes y de defunciones $(p=0.01)$ en la totalidad de los casos. Discusión y conclusiones: Las variaciones en los niveles de urea, creatinina y plaquetas en los pacientes con ECMO pueden tener mayor peso en la toma de decisión clínica.
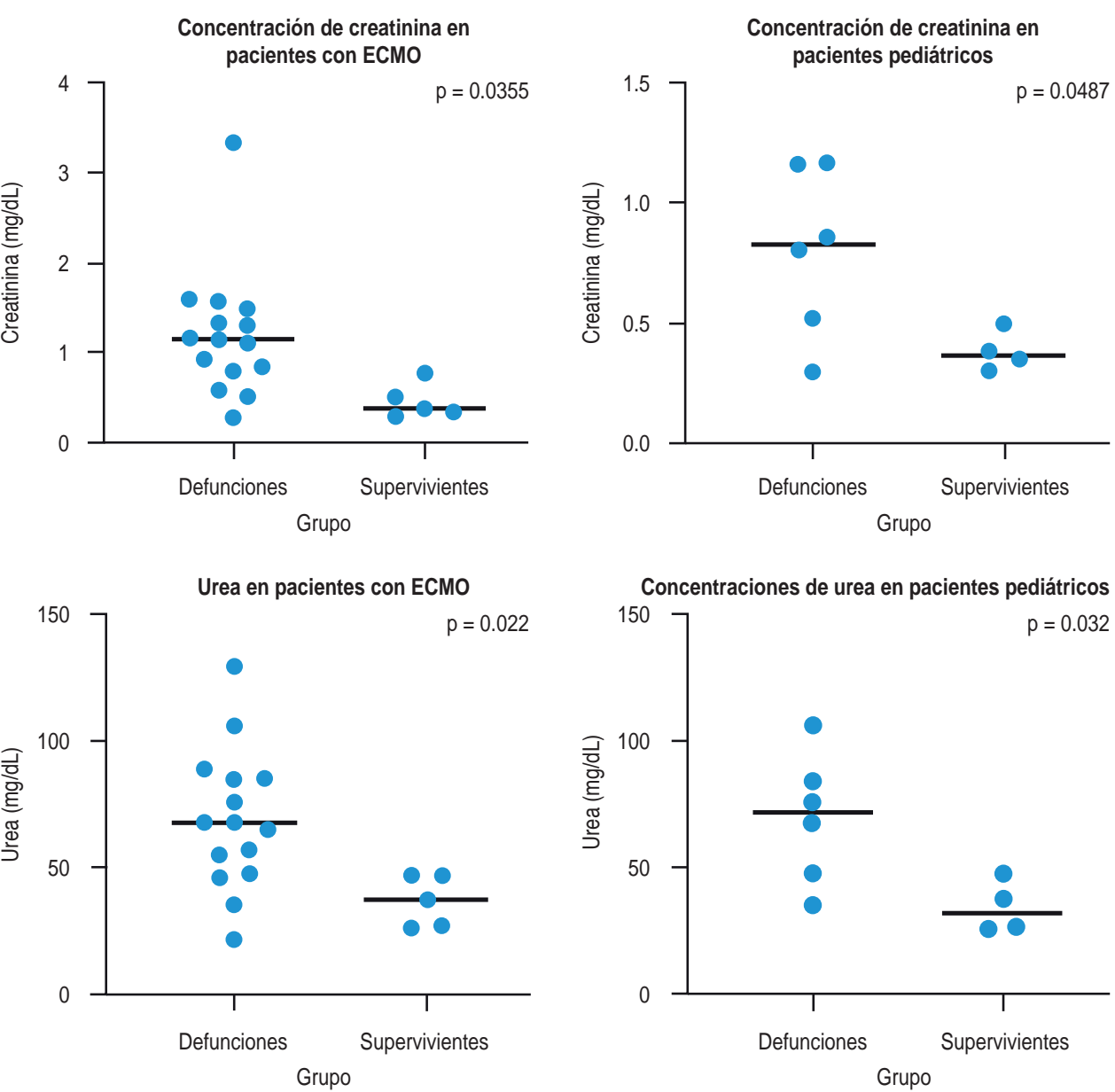

Figura 9.4.1:

Comparación de medidas analíticas en pacientes con ECMO en el grupo de defunciones comparado con el grupo de supervivientes.
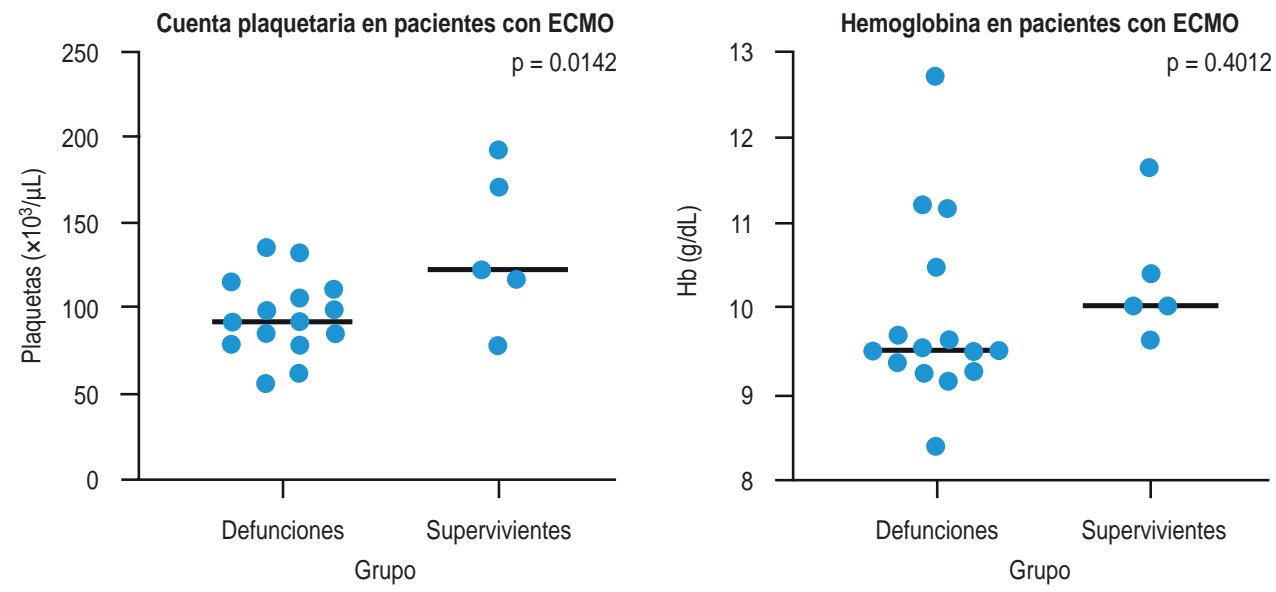
Tabla 9.4.1: Características bioquímicas y hematológicas de pacientes sometidos a terapia con membrana de oxigenación extracorpórea (ECMO).

\begin{tabular}{|c|c|c|c|c|c|c|c|c|c|}
\hline \multirow[b]{2}{*}{ Analito } & \multicolumn{3}{|c|}{ General } & \multicolumn{3}{|c|}{ Pacientes pediátricos } & \multicolumn{3}{|c|}{ Pacientes adultos } \\
\hline & $\begin{array}{l}\text { Supervivientes } \\
(\mathrm{n}=9)\end{array}$ & $\begin{array}{l}\text { Defunciones } \\
\quad(\mathrm{n}=11)\end{array}$ & $\mathbf{p}$ & $\begin{array}{l}\text { Supervivientes } \\
(\mathrm{n}=4)\end{array}$ & $\begin{array}{l}\text { Defunciones } \\
\qquad(\mathrm{n}=6)\end{array}$ & $\mathbf{p}$ & $\begin{array}{c}\text { Supervivientes } \\
(\mathbf{n}=1)\end{array}$ & $\begin{array}{l}\text { Defunciones } \\
\qquad(\mathrm{n}=9)\end{array}$ & p \\
\hline Hemoglobina (g/dL) & $10.35 \pm 0.78$ & $9.90 \pm 1.08$ & 0.4012 & $10.53 \pm 0.77$ & $10.48 \pm 1.32$ & 0.9414 & 9.63 & $9.52 \pm 0.73$ & N/A \\
\hline Plaquetas $\left(\times 10^{3} / \mu \mathrm{L}\right)$ & $136.42 \pm 45.39$ & $95.50 \pm 22.50$ & 0.0142 & $127.83 \pm 47.49$ & $90.89 \pm 29.07$ & 0.1612 & 170.76 & $98.57 \pm 18.19$ & N/A \\
\hline $\mathrm{TP}(\mathrm{sst})$ & $4.65 \pm 2.45$ & $6.82 \pm 5.95$ & 0.4443 & $5.32 \pm 2.25$ & $8.51 \pm 9.06$ & 0.5164 & 2 & $5.69 \pm 2.64$ & N/A \\
\hline TTPa (sst) & $22.99 \pm 9.49$ & $36.92 \pm 15.32$ & 0.0741 & $24.66 \pm 10.07$ & $36.67 \pm 12.4$ & 0.0795 & 16.32 & $35.08 \pm 17.45$ & N/A \\
\hline Creatinina (mg/dL) & $0.47 \pm 0.19$ & $1.20 \pm 0.70$ & 0.0355 & $0.39 \pm 0.08$ & $0.80 \pm 0.35$ & 0.0487 & 0.79 & $1.47 \pm 0.77$ & N/A \\
\hline Urea (mg/dL) & $36.89 \pm 10.09$ & $68.97 \pm 27.57$ & 0.0220 & $34.48 \pm 9.85$ & $69.56 \pm 25.39$ & 0.032 & 46.54 & $68.57 \pm 30.44$ & N/A \\
\hline Albúmina (g/dL) & $3.82 \pm 0.69$ & $3.33 \pm 0.62$ & 0.1617 & $3.91 \pm 0.76$ & $3.91 \pm 0.32$ & 0.985 & 3.46 & $2.95 \pm 0.45$ & N/A \\
\hline AST (U/L) & $116.62 \pm 103.82$ & $535.09 \pm 873.17$ & 0.3075 & $138.67 \pm 105.50$ & $870.66 \pm 1,325.54$ & 0.3116 & 28.4 & $311.38 \pm 308.75$ & N/A \\
\hline $\operatorname{ALT}(\mathrm{U} / \mathrm{L})$ & $39.50 \pm 31.08$ & $214.17 \pm 284.73$ & 0.1954 & $46.43 \pm 31.11$ & $348.30 \pm 397.06$ & 0.1753 & 11.8 & $124.75 \pm 144.40$ & N/A \\
\hline
\end{tabular}

sst = segundos sobre el testigo; $\mathrm{TP}$ = tiempo de protrombina; TTPa = tiempo de tromboplastina parcial activada; AST = aspartato aminotransferasa; ALT = alanino aminotransferasa .

\subsection{Uso de vancomicina en esternotomía como prevención para mediastinitis en cirugía cardiaca}

Cuevas Álvarez Roberto,* Hernández Hernández Francisco Javier,* Álvarez Moreno Carolina del Carmen, ${ }^{\ddagger}$ Hernández Trejo Luis León,* Olvera Tizoc Jorge,* Ramírez Castañeda Alberto,* Riera Kinkel Carlos* * Hospital de Cardiología del Centro Médico Nacional Siglo XXI. ‡ Unidad Médica de Alta Especialidad No. 1 Bajío del IMSS. Tipo de estudio: Estudio terapéutico

Introducción: La infección en sitio quirúrgico es una complicación poco frecuente, en cirugía cardiovascular, con una prevalencia de 0.3-4.4\%, múltiples intervenciones se realizan como parte del protocolo de prevención, la administración de vancomicina tópica en el hueso esponjoso esternal ha sido reportada en la literatura reciente con resultados mixtos. Objetivo: Evaluar la administración tópica de vancomicina en la prevención de infección en sitio quirúrgico en la cirugía cardiaca por estereotomía media. Metodología: Se colocó aleatoriamente pasta de vancomicina a pacientes sometidos a cirugía cardiaca en el periodo comprendido de octubre 2017 a octubre 2018 versus pacientes en los que se aplicó cera ósea de

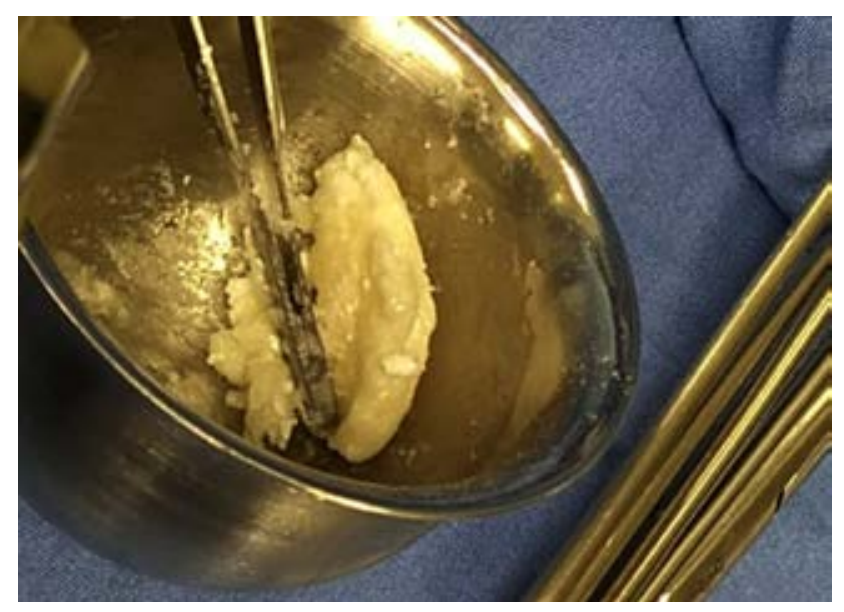

Figura 9.5.1.

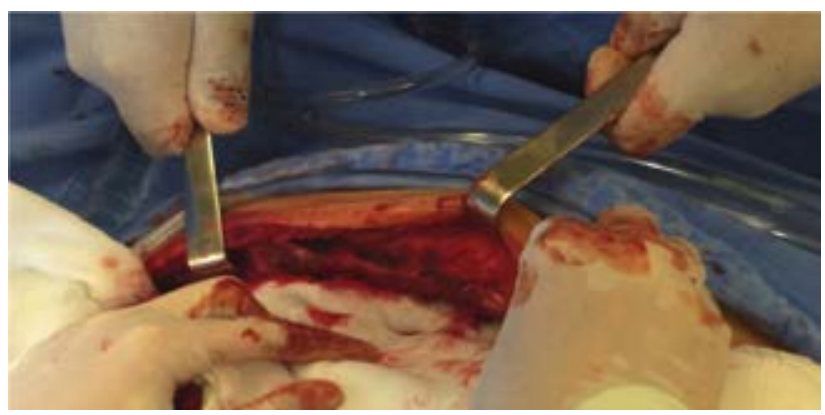

Figura 9.5.2.

manera convencional. Resultados: Se realizaron 869 cirugías en total, 30 (3.45\%) pacientes sufrieron infecciones de sitio quirúrgico en los que se aplicó cera ósea. De los 64 pacientes (7.36\%) a los que se aplicó pasta de vancomicina, un paciente $(0.11 \%)$ presentó infección de herida superficial. Discusión y conclusiones: La administración tópica de vancomicina en la porción esponjosa del esternón es eficaz en disminuir la prevalencia de la infección en sitio quirúrgico en los pacientes con cirugía.

\subsection{Aneurisma ventricular izquierdo congénito: reporte de caso}

Navarro Jorge Luis

Hospital de Cardiología del Centro Médico Nacional Siglo XXI.

Tipo de estudio: Reporte de casos clínicos con revisión de fuentes

Introducción: El aneurisma ventricular es una zona discinética con una conexión ancha al ventrículo, asintomático o con eventos embólicos, falla cardiaca y muerte súbita. La etiología es la cardiopatía isquémica. El AV congénito es muy rara, con una prevalencia de $0.04 \%$, con una media de edad de 31.5 años. Descripción del caso: Mujer de 33 años sin antecedentes de importancia. Inició con palpitaciones en 2012, documentando aumento del automatismo ventricular. Tuvo tratamiento con propafenona durante dos meses. Asintomática hasta 2017 cuando presentó disnea y palpitaciones, por lo cual acude a nuestro hospital. Se realizó Holter que evi- 


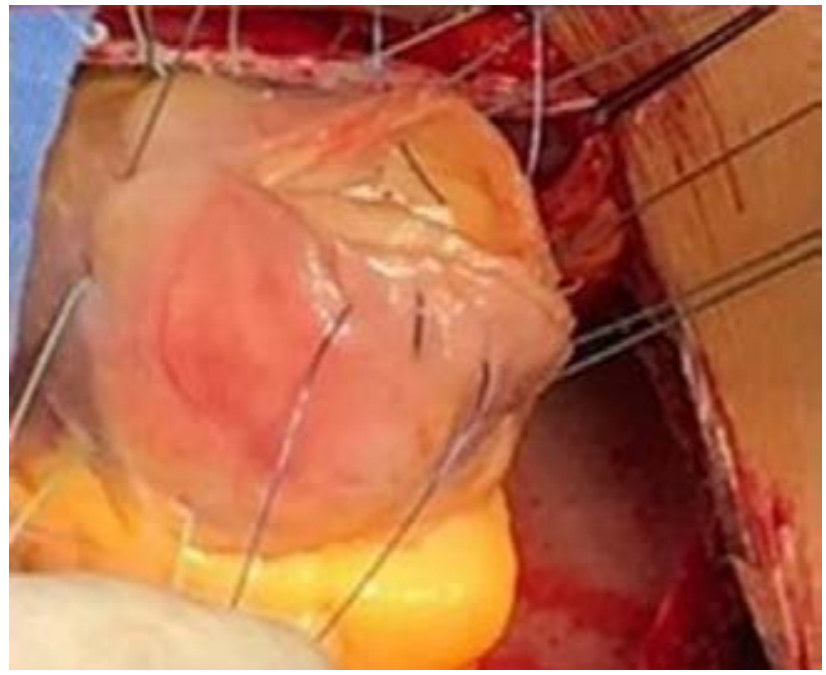

Figura 9.6.1.

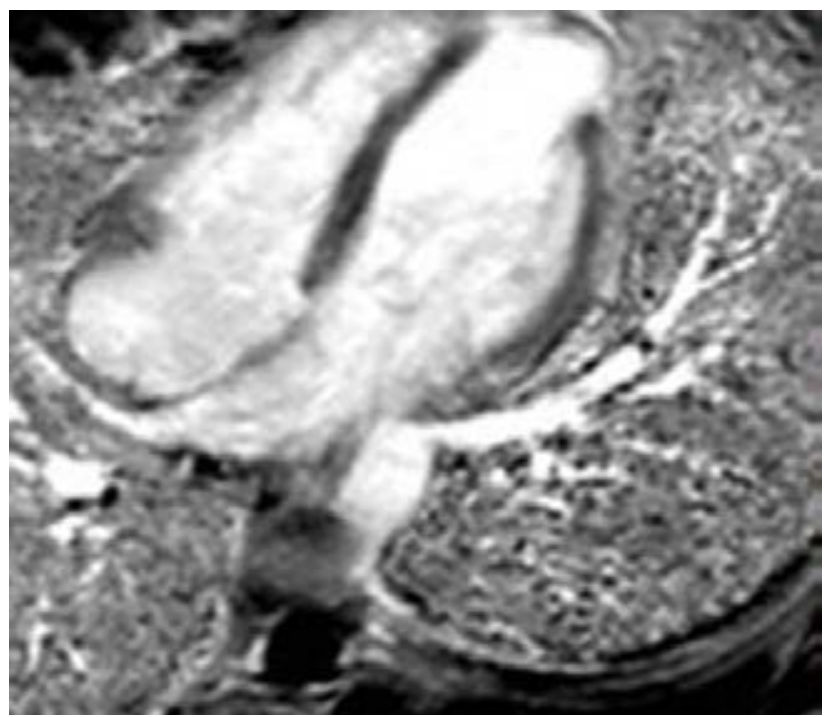

Figura 9.6.2.

denció 110 episodios de taquicardia ventricular no sostenida. La resonancia magnética demostró AV apical ventricular izquierdo. Metodología: En este reporte de caso mostramos un resumen del manejo quirúrgico de aneurisma ventricular en nuestra institución y la evolución del mismo. También mostramos un panorama general del manejo de aneurisma ventricular. Resultados: Se diagnosticó con AV congénito y se propuso para aneurismectomía, la cual se realizó en julio de 2018. La serología para enfermedad de Chagas y perfil inmunológico se reportaron negativos. La evolución postquirúrgica fue favorable, siendo egresada al tercer día posterior a la cirugía de la Unidad de Cuidados Intensivos Postquirúrgicos. A los seis meses de seguimiento se mantiene asintomática. Discusión: El tratamiento quirúrgico del AVI sintomático ha demostrado mejorar la esperanza y la calidad de vida de los enfermos, con una mortalidad operatoria baja y en descenso. En este reporte de caso mostramos que en nuestra institución se realizó la corrección quirúrgica y el paciente tuvo una adecuada evolución.

\section{Trabajo ganador del premio Dr. Carlos Pérez Treviño Tercer Lugar}

\section{7. ¿Cuál es el rendimiento del modelo EuroSCORE II en nuestro medio?}

Teniente Valente Raúl, ${ }^{*}$ Martínez Bautista Humberto, ${ }^{\ddagger}$ Chagolla Santillán Miguel Ángel,* Acevedo Bañuelos Iliana,* García Muñoz Iván,* Romo Escamilla Ricardo Elías*

* Hospital Regional de Alta Especialidad del Bajío. ${ }^{\ddagger}$ Centro de Investigaciones en Matemáticas (CIMAT). Tipo de estudio: Estudio pronóstico

Introducción: El modelo pronóstico EuroSCORE para mortalidad perioperatoria en cirugía cardiaca publicado en 1999 ha sido utilizado ampliamente en diversas latitudes. En los últimos lustros mostró deficiencias en su calibración aunque siguió conservando buena discriminación. El modelo fue actualizado y publicado en el 2012 y fue llamado EuroSCORE II. Objetivo: Evaluar el desempeño del EuroSCORE II en pacientes sometidos a cirugía cardiaca con y sin bomba de circulación extracorpórea en el Hospital Regional de Alta Especialidad del Bajío (HRAEB) de León, Guanajuato. Metodología: Estudio observacional, transversal, retrospectivo, pacientes $\geq 16$ años operados del 01/01/2008 al 31/12/2013. Se registró la mortalidad hospitalaria y se calculó el EuroSCORE II. Se valoró la discriminación por el área bajo la curva ROC. La calibración fue evaluada mediante la prueba $\chi^{2}$ de bondad de ajuste de Hosmer-Lemeshow $(\mathrm{H}-\mathrm{L})$ y la razón de mortalidad ajustada al riesgo (RAMR). Resultados: 338 pacientes, edad $49.87 \pm 16.61$ años (16-80), 176 hombres $(52.1 \%)$ y 162 mujeres (47.9\%). Tipos de cirugía: valvular 110 (32.2\%), revascularización 102 (29.8\%), congénitos 52 (15.2\%) y otros 78 (22.8\%). El EuroSCORE II fue de $4.1 \pm 5.3$. La mortalidad observada fue de 10.9\%. El área bajo la curva ROC fue de 0.806 (IC 95\% 0.739-0.872) compatible con buena discriminación. La $\chi^{2}$ de H-L fue 13.2 con $p$ $=0.104$, sugestiva de calibración adecuada. La RAMR fue de 2.65, compatible con infraestimación global del modelo, principalmente en los deciles de alto riesgo. Discusión y conclusiones: EuroSCORE II es confiable para estimar las probabilidades de muerte en pacientes en el HRAEB. Buena discriminación. Calibración global adecuada pero el RAMR sugiere infraestimación del riesgo. 